\title{
Evaluación de Polietileno Maleatado en Compuestos de Etil Vinil Acetato y Harina Telinne Monspessulana
}

\author{
Oscar Palacio*, Oscar Y. Buitrago y Arnoldo E. Delgado \\ Facultad de Ingeniería, Universidad Militar Nueva Granada, Carrera 11 No. 101-80, Bogotá, Colombia. \\ (e-mail: oscar.palacio@unimilitar.edu.co; oscar.buitrago@unimilitar.edu.co; \\ arnoldo.delgado@unimilitar.edu.co) \\ * Autor a quien debe ser dirigida la correspondencia
}

Recibido Jul. 29, 2015; Aceptado Sep. 29, 2015; Versión final Oct. 31, 2015, Publicado Feb. 2016

\begin{abstract}
Resumen
Se investigó el efecto de la adición de polietileno maleatado en compuestos de etilen vinil acetato reforzados con harina de Telinne Monspessulana previamente mercerizada. Dos factores fueron analizados: (i) concentración del agente de acoplamiento con cinco niveles 2, 4, 6, 8, 10 partes por cien de polímero; y (ii) concentración de harina con dos niveles: 25 y 40 partes por cien de polímero. El efecto del polietileno maleatado sobre la compatibilidad entre etilen vinil acetato y la harina vegetal fue evaluado a través de ensayos de tensión a los compuestos. Los análisis permiten establecer que el agente de acoplamiento tipo polietileno maleatado mejoró la compatibilidad de los compuestos, efecto que fue evidenciado en los valores del módulo de Young y la resistencia a la tracción. Con base en estos resultados se concluye que el polietileno maleatado es una alternativa como agente de acoplamiento en compuestos de etilen vinil acetato y fibras naturales.
\end{abstract} acoplamiento

\section{Evaluation of Maleated Polyethylene in Ethylene Vinyl Acetate and Telinne Monspessulana Flour Composites}

\begin{abstract}
The effect of the addition of maleated polyethylene to compounds of ethylene vinyl acetate with Telinne Monspessulana flour previously mercerized was investigated. Two factors were analyzed: (ii) concentration of maleated polyethylene with five levels $2 ; 4 ; 6 ; 8 ; 10$ parts per hundred polymer; and (ii) concentration of flour with two levels 25 and 40 parts per hundred of polymer. The effect of maleated polyethylene on compatibility between ethylene vinyl acetate and flour was evaluated by tensile testing of compounds. Analysis indicates that the coupling agent improved the compatibility between Telinne Monspessulana flour and ethylene vinyl acetate, effect that was observed by the values of tensile strength and Young's modulus. Based on these results, maleated polyethylene is a good alternative as a coupling agent in ethylene vinyl acetate and natural fibers compounds.
\end{abstract}

Keywords: ethylene vinyl acetate; Telinne Monspessulana; tensile properties; coupling agent 


\section{INTRODUCCIÓN}

En décadas recientes el uso de fibras naturales como cargas reforzantes en materiales compuestos ha tomado cada vez mayor auge debido a la preocupación por el desarrollo sostenible de compuestos poliméricos ambientalmente amigables y de bajo costo. Aunque las fibras celulósicas tienen ventajas como la biodegradabilidad, sostenibilidad y baja densidad (Pracella et al., 2010; Malunka et al., 2006; Rodriguez et al., 2014; Solis et al., 2005; Zimmermann et al., 2014); su naturaleza hidrofilica provoca una pobre adhesión en matrices no polares, causando una disminución en el rendimiento mecánico del material. Lograr una alta dispersión de la carga reforzante y alcanzar una adhesión interfacial adecuada que permita la transferencia de esfuerzos al interior del compuesto (Dikobe y Luyt, 2010; Madera-Santana et al. 2013), es posible a través de la modificación de la superficie de la fibra por medio de tratamientos químicos tales como la mercerización, acetilación, tratamiento con silanos o maleatos, etc. que permitan reducir su carácter hidrofilico o el uso de agentes de acoplamiento que mejoren su interacción con la matriz del polímero (Faruk et al., 2012; Kabir et al. 2012; Keener et al., 2004; Li et al., 2007; Yang et al., 2007).

Estudios sobre el uso de agentes de acoplamiento en compuestos de etilen vinil acetato (EVA), reportan que su uso reduce drásticamente la tasa de coalescencia y disminuye el tamaño de los dominios como resultado de la disminución de la tensión interfacial proporcionada por el uso del compatibilizador (Bakar et al., 2015; Bhattacharyya et al., 2005; Bhattacharyya et al., 2001; Ma et al., 2012; Radhakrishnan et al., 2007). (Dikobe y Luyt, 2007), indican en su investigación, que el módulo de Young de compuestos de EVA/ harina de madera aumentan con el incremento del contenido de poli metacrilato de etileno-co-glicidilo, como resultado del aumento de la interacción interfacial entre la matriz y la fase dispersa. (Bakar et al., 2015), encontraron que en mezclas de Policloruro de Vinilo /EVA con fibras de kenaf, el uso de polimetil metacrilato como agente de acoplamiento mejora la adhesión interfacial entre la fibra y la matriz, mejorando la estabilidad térmica del material. (Madera-Santana et al., 2013) establecen que el tratamiento con silanos en compuestos de EVA reforzados con fibras de sisal, provee buenas propiedades de tracción, debido a su superficie áspera y el enlace químico entra la fibra y la matriz de EVA.

Si bien el uso de fibras naturales como cargas reforzantes en matrices poliméricas proporciona mayor rigidez al compuesto (Elanthikkal et al., 2013; Malunka et al., 2006; Silviya et al., 2012; Sonia et al., 2013), en la elaboración de materiales termoplásticos de EVA, se tiene la alternativa de reticular las cadenas poliméricas para conferirle mayor resistencia mecánica al material, pero el proceso de acoplamiento de la fibra con el polímero puede verse afectado por la aparición de reacciones secundarias como la reticulación (Hwang et al., 2012; Kim et al., 2001; Yin et al., 2006) .

En este estudio, se analizó el efecto del agente de acoplamiento polietileno maleatado (MAPE) en compuestos de etilen vinil acetato (EVA) reforzados con harina de Telinne Monspessulanna (HTM). La mezcla se realizó en un mezclador interno y las probetas fueron obtenidas por medio de moldeo por compresión. La compatibilidad de las mezclas EVA/HTM/MAPE fue evaluada por medio de la determinación de sus propiedades mecánicas.

\section{MATERIALES Y MÉTODOS}

Materiales: HTM con diámetro de particula 100-800 $\mu \mathrm{m}$ y humedad $0.50 \%$. EVA, Elvax $460^{\circledR}$ fabricado por Dupont ${ }^{\mathrm{TM}}$. Polietileno maleatado MAPE, tasa de injerto 0.9 , densidad $0.95 \mathrm{~g} / \mathrm{cm} 3$, comercializado por Hangzhou Uniwise ${ }^{\circledR}$ Internacional Co. Ltd. Struktol WB $16^{\circledR}$, fabricado por Struktol ${ }^{\circledR}$. Irganox 1076 fabricado por BASF ${ }^{\mathrm{TM}}$. Hidróxido de sodio con $98 \%$ pureza; ácido acético glacial, pureza of $99.6 \%$; agua desionizada, rango de conductividad entre $3.0-6.5 \mu \mathrm{S} / \mathrm{cm}$.

Diseño experimental. Se diseñó un experimento bifactorial 2x6 completamente al azar. Factor A: HTM con dos niveles 25 y 40 php. Factor B: MAPE con seis niveles 0; 2; 4; 6; 8 y 10 php; para un total de doce formulaciones. La suma EVA + MAPE fue llevada a 100 partes (php) y se tomó como referencia para cuantificar los ingredientes, según se observa en la Tabla 1. Antioxidante y ayuda de proceso permanecieron constantes en todas las formulas. La variable de salida fue asignada a cada una de las propiedades mecánicas del compuesto, se realizaron 5 repeticiones por variable.

Análisis estadístico. La influencia del MAPE sobre las propiedades mecánicas de WPC fue analizada aplicando ANOVA de dos factores con el programa Statgraphics ${ }^{\circledR 5} .0$ usando nivel de confianza del $95 \%$. La prueba de comparación de medias se realizó aplicando Tukey HSD.

Mercerización de HTM. Se sumergieron $500 \mathrm{~g}$ de HTM en 10 litros de solución de hidróxido de sodio al 8\% durante 4 horas a $35{ }^{\circ} \mathrm{C}$ con agitación constante. Después se lavó con disolución de ácido acético al $1 \%$ hasta que la solución de lavado tuvo pH de 6.8. Posteriormente fue secada en un horno de convección forzada a $95{ }^{\circ} \mathrm{C}$ durante 24 horas, el contenido final de humedad fue $0.5 \%$. 
Tabla 1: Formulaciones. Todas las cantidades en php

\begin{tabular}{|c|l|c|c|c|c|c|c|}
\hline (A) Niveles de & Ingredientes & \multicolumn{6}{|c|}{ (B) Niveles de MAPE } \\
\hline HTM & & 0 & 2 & 4 & 6 & 8 & 10 \\
\hline \multirow{3}{*}{40} & EVA & 100 & 98 & 96 & 94 & 92 & 90 \\
\cline { 2 - 8 } & Antioxidante & 1.0 & 1.0 & 1.0 & 1.0 & 1.0 & 1.0 \\
\cline { 2 - 8 } & Ayuda de proceso & 1.0 & 1.0 & 1.0 & 1.0 & 1.0 & 1.0 \\
\hline \multirow{3}{*}{25} & EVA & 100 & 98 & 96 & 94 & 92 & 90 \\
\cline { 2 - 8 } & Antioxidante & 1.0 & 1.0 & 1.0 & 1.0 & 1.0 & 1.0 \\
\cline { 2 - 8 } & Ayuda de proceso & 1.0 & 1.0 & 1.0 & 1.0 & 1.0 & 1.0 \\
\hline
\end{tabular}

Mezclado. La elaboración de la mezcla se realizó en un mezclador interno Ml 60 formado por 2 rodillos triangulares, con torque máximo de $10 \mathrm{~N} / \mathrm{m}$ y capacidad de $60 \mathrm{~cm}^{3}$. El orden de adición fue el siguiente, primero se adicionó el EVA, en seguida el MAPE, posteriormente la fibra mercerizada, al finalizar la etapa de incorporación se agregó la ayuda de proceso. La temperatura de la mezcla fue $120 \pm 5^{\circ} \mathrm{C}$ y el tiempo total de mezclado fue 10 minutos.

Prensado. Las láminas fueron obtenidas por medio de moldeo por compresión, este procedimiento es de común uso en la elaboración de probetas a nivel de laboratorio (Navia et al., 2015). El compuesto fue colocado en un molde con dimensiones $16 \mathrm{~cm} \times 16 \mathrm{~cm}$ con espesor de $3.45 \mathrm{~mm}$. Se utilizó una prensa hidráulica con sistema de calefacción por medio de resistencia eléctrica. Los parámetros de operación fueron: Temperatura del molde $160 \pm 1^{\circ} \mathrm{C}$, presión especifica de $5.24 \mathrm{MPa}$, tiempo de prensado 6 minutos.

Ensayos de Tensión. Las probetas fueron obtenidas mediante el proceso de troquelado, con geometría tipo I según la norma ASTM D638-10. El ensayo de tensión fue realizado en maquina universal Shimadzu ${ }^{\circledR}$ AGSX. Parámetros del ensayo: velocidad de desplazamiento $10 \mathrm{~mm} / \mathrm{min}$, temperatura $24{ }^{\circ} \mathrm{C}$ y humedad relativa $45 \%$. Se emplearon cinco (5) probetas por cada prueba.

Determinación de la Dureza. Se realizó según la norma ASTM D 2240-05. El equipo utilizado fue el durómetro INSIZE ${ }^{\circledR}$ tipo D. Cinco (5) mediciones fueron efectuadas por formula.

\section{RESULTADOS Y DISCUSIÓN}

\section{Módulo de Young}

El módulo de Young del WPC está formado por el aporte de los módulos del EVA, HTM y MAPE. El módulo del EVA con $18 \%$ de acetato de vinilo es aproximadamente $52 \mathrm{MPa}$, mientras que la harina de madera tiene un módulo cercano a $2500 \mathrm{MPa}$. Como se puede apreciar en la Figura 1, cuando se incrementa la cantidad de carga reforzante, la concentración de MAPE no tiene gran influencia sobre el módulo de Young de los WPC.

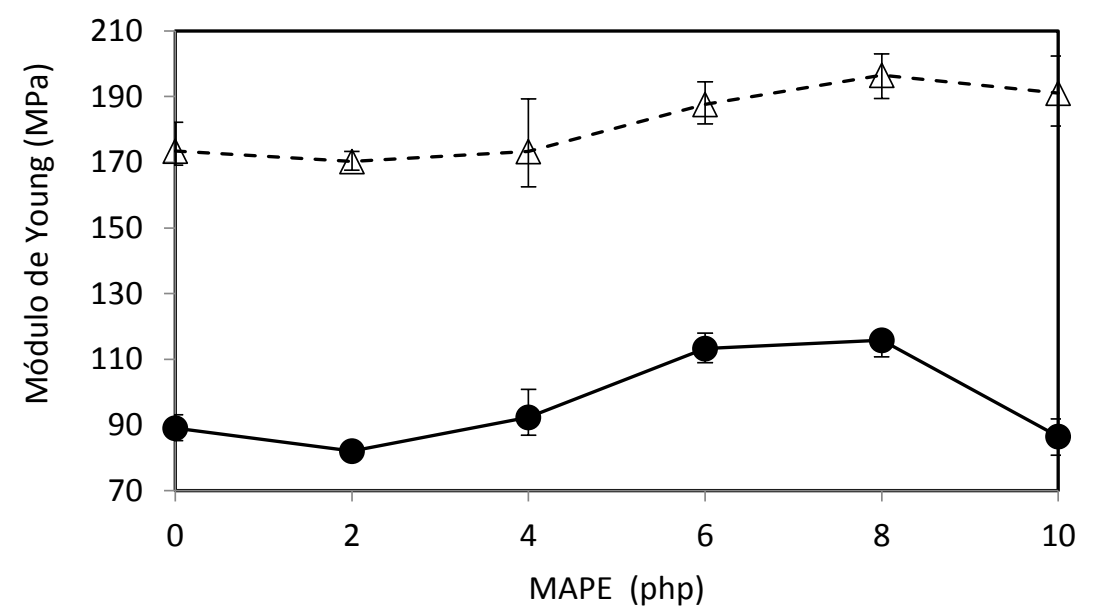

Fig.1: Compuesto de EVA. Módulo de Young. - - $\Delta$ - - HTM 40 php $\quad$-•- HTM 25 php 
Evidentemente, el factor que influye de manera significativa sobre esta propiedad es el nivel de carga. Se puede observar claramente que a mayor cantidad de HTM, el módulo de las formulaciones aumenta, como resultado del incremento en la rigidez de las probetas. Para cualquier nivel del MAPE, el módulo es superior para los WPC con 40 php de carga, con respecto a los compuestos elaborados con 25 php de HTM aproximadamente en un $65 \%$. Resultado acorde con varias investigaciones, en donde se reporta el aumento en el módulo de elasticidad de compuestos poliméricos reforzados con fibras naturales (Arif et al., 2009; Bengtsson y Oksman, 2006; Salmah et al., 2011).

Para 40 php de HTM, se observa leve aumento en el módulo de las formulaciones con 8 php de MAPE, resultado ligeramente superior con respecto al compuesto sin agente de acoplamiento en un $13 \%$. Con respecto al compuesto de EVA elaborado con 25 php de carga, se encontró un incremento en las mezclas con 6 y 8 php de MAPE de aproximadamente $28 \%$ con respecto a las formulaciones sin AA.

\section{Límite de fluencia}

En la Figura 2, se puede observar como el esfuerzo de fluencia es mayor para los compuestos elaborados con 40 php de HTM con respecto a las mezclas con 25 php de carga, para todos los niveles de MAPE. Al analizar la dispersión de los datos, es claro que la concentración de MAPE, no influye significativamente sobre el esfuerzo de fluencia de los compuestos con 40 php de HTM, caso opuesto al observado en las formulaciones con 25 php de harina.

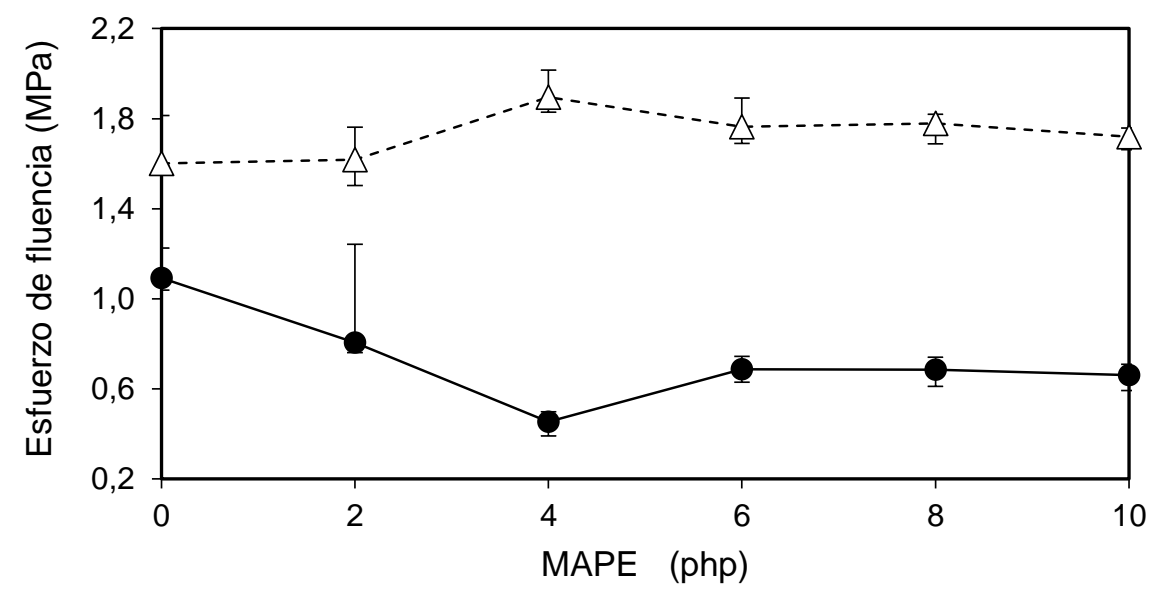

Fig. 2: Compuesto de EVA. Esfuerzo de fluencia. - - - - - HTM 40 php $\quad-\bullet-$ HTM 25 php.

Con respecto a la deformación elástica, como se puede apreciar en la Figura 3, es afectado tanto por la concentración de MAPE como por el nivel de carga. Para los compuestos con 40 php de HTM, se presentó un incremento notable entre 2 y 4 php de MAPE. En los compuestos de EVA con 25 php de carga, la incorporación del agente de acoplamiento disminuye el esfuerzo de fluencia en comparación con las formulaciones sin MAPE.

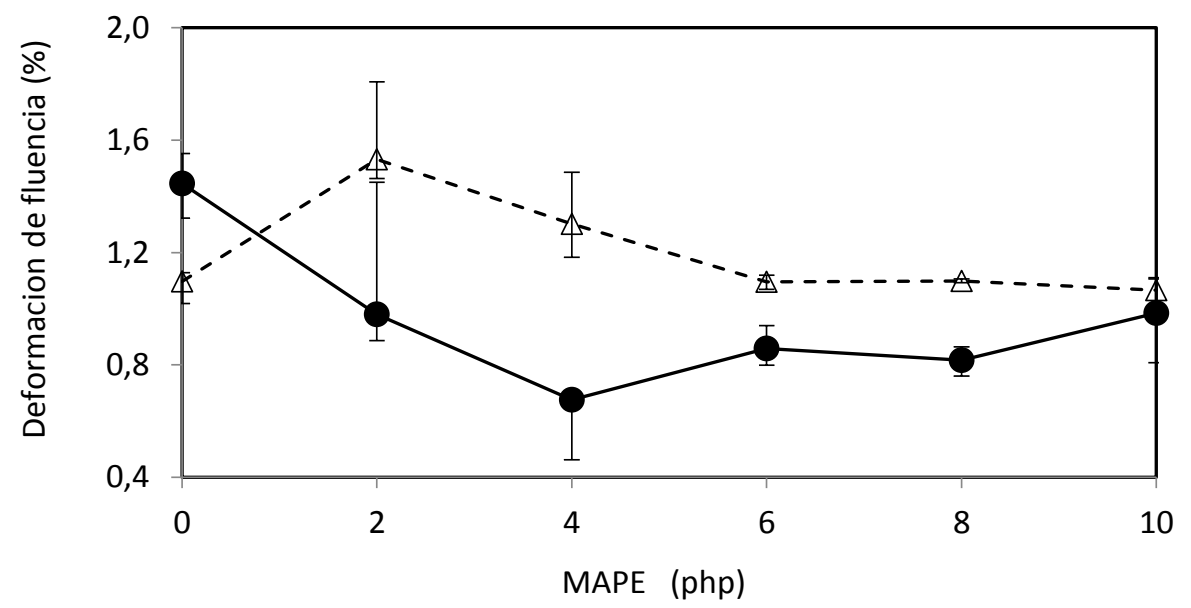

Fig. 3: Compuesto de EVA. Deformación de fluencia. - - $\Delta$ - - HTM 40 php $\quad-\bullet-$ HTM 25 php. 
En otras palabras, el Límite de fluencia de los compuestos con 40 php no presentó variación significativa, por lo tanto, no se puede afirmar categóricamente que la concentración de MAPE tenga incidencia. Sin embargo, queda claro que el límite de fluencia es superior en las mezclas con $40 \mathrm{php}$ de HTM y que la harina es la principal responsable del aumento de la propiedad.

\section{Resistencia a la tracción}

Los compuestos elaborados con 40 php de carga presentan mayor resistencia a la tracción en comparación con los WPC con 25 php de HTM, excepto en el ensayo con cero MAPE, donde estadísticamente no hay diferencia entre estos valores (ver Figura 4). Claramente la adición de MAPE mejora significativamente la tracción en ambos niveles de harina (25 y 40 php), esto gracias a la acción compatibilizante del AA la cual permite que la trasferencia de esfuerzos al interior del material sea muchos más efectiva y por ende el material puede soportar mayores tensiones (Onuegbu et al., 2012).

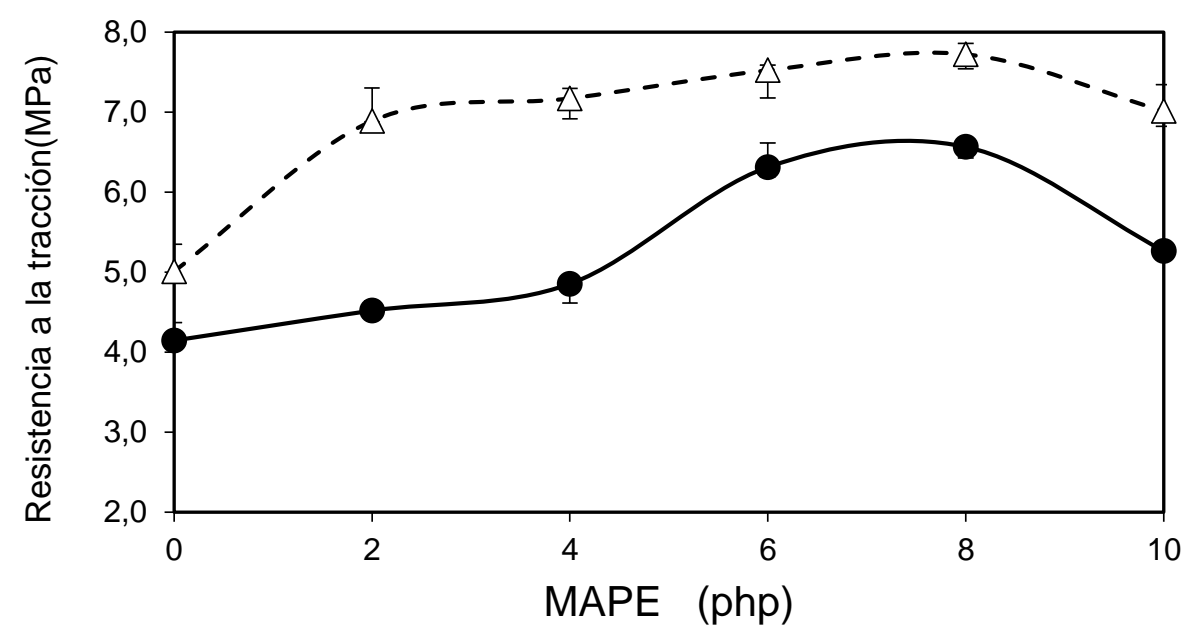

Fig. 4: Compuesto de EVA. Resistencia a la tracción. - - $\Delta$ - - HTM 40 php $\quad-\bullet-$ HTM 25 php.

Por otro lado, dada la polaridad de los grupos acetato de vinilo en el EVA, estos monómeros puede contribuir para que la adhesión interfacial entre la harina y la matriz polimérica sea más eficaz al interactuar con los grupos hidroxilo de la celulosa en la HTM, logrando así, mayor adherencia de la superficie harinapolímero. Tanto en los compuestos de 25 como 40 php de HTM, se obtuvo un máximo en la resistencia a la tracción en las formulaciones con 8 php de MAPE. Se alcanzaron incrementos del 58 y $48 \%$ con respecto al compuesto sin MAPE, para 25 y 40 php de carga respectivamente. Un aumento ulterior a 8 php de MAPE ocasiona la disminución de la resistencia a la tracción, indistintamente del contenido de carga.

\section{Deformación a rotura}

La figura 5 muestra claramente como la deformación a la rotura de los compuestos disminuye con el aumento del nivel de carga. Como se puede observar, los compuestos de EVA con 25 php de HTM, poseen mayor elongación con respecto a aquellos que tienen 40 php de harina, hecho que se puede atribuir a que en la formulación hay mayor cantidad de polímero base y sus propiedades intrínsecas se manifiestan con mayor facilidad en el compuesto; además al aumentar el contenido de carga, se reduce el volumen libre y puede que la propagación de las tensiones no sea tan eficaz, dada la mayor probabilidad de encontrar sitios de falla en la interfaz harina - polímero.

En relación al nivel de agente de acoplamiento MAPE, es claro como la elongación de los compuestos de EVA sin agente de acoplamiento, es superior con respecto a las mezclas que lo contienen. El acoplamiento de la harina con la matriz polimérica, restringe la movilidad molecular, dificultando el desplazamiento de las cadenas en el polímero, razón por la cual se observa una disminución de la deformación a rotura de los WPC al incrementar el contenido de MAPE en las formulaciones. La baja deformación a rotura de los compuestos EVA/HTM/MAPE, también puede estar influenciada por problemas de compatibilidad entre el EVA y el MAPE, pero esta condición se encuentra fuera de los alcances de la presente investigación.

\section{Dureza}

La adición del agente de acoplamiento, aumentó la dureza de los compuestos de EVA independientemente del contenido de carga (Bakar et al., 2014), como se puede apreciar en la Figura 4. Para concentraciones de MAPE iguales o superiores a 4 php, las durezas de los compuestos son superiores en comparación con 
la formulación sin agente de acoplamiento, indistintamente del nivel de HTM. Caso particular se presenta con las formulaciones con 10 php de MAPE en donde las durezas de las mezclas con 25 y 40 php de HTM, son muy similares.

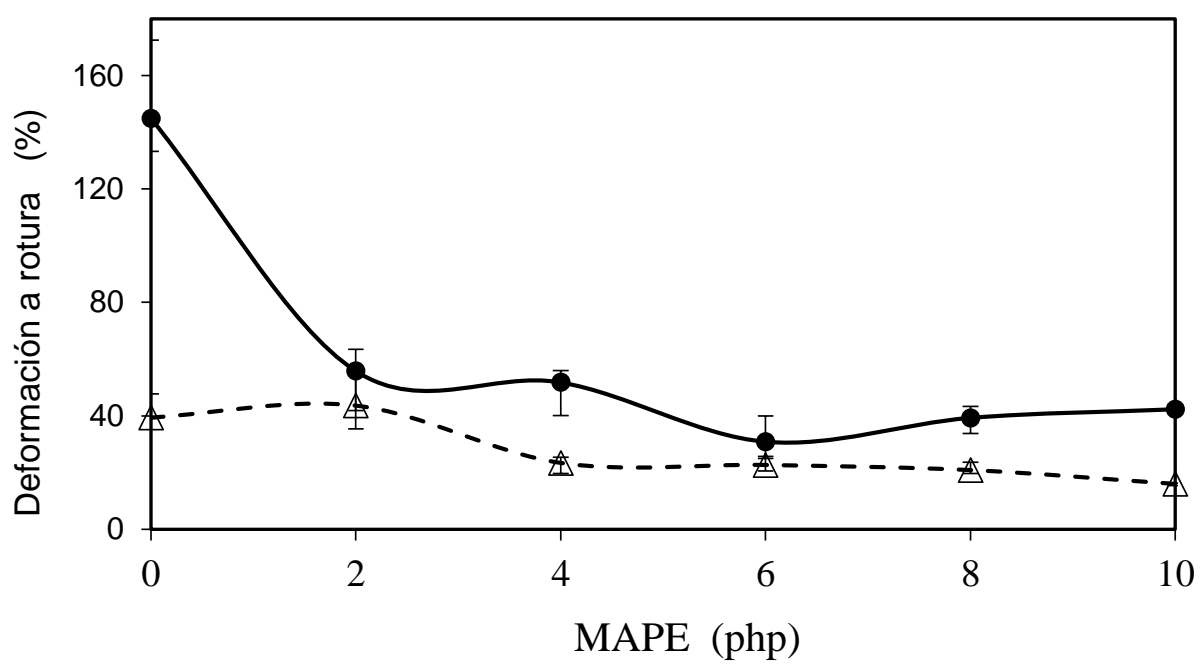

Fig. 5: Compuesto de EVA. Deformación a rotura. - - $\Delta-$ - HTM 40 php $\quad-\bullet-$ HTM 25 php.

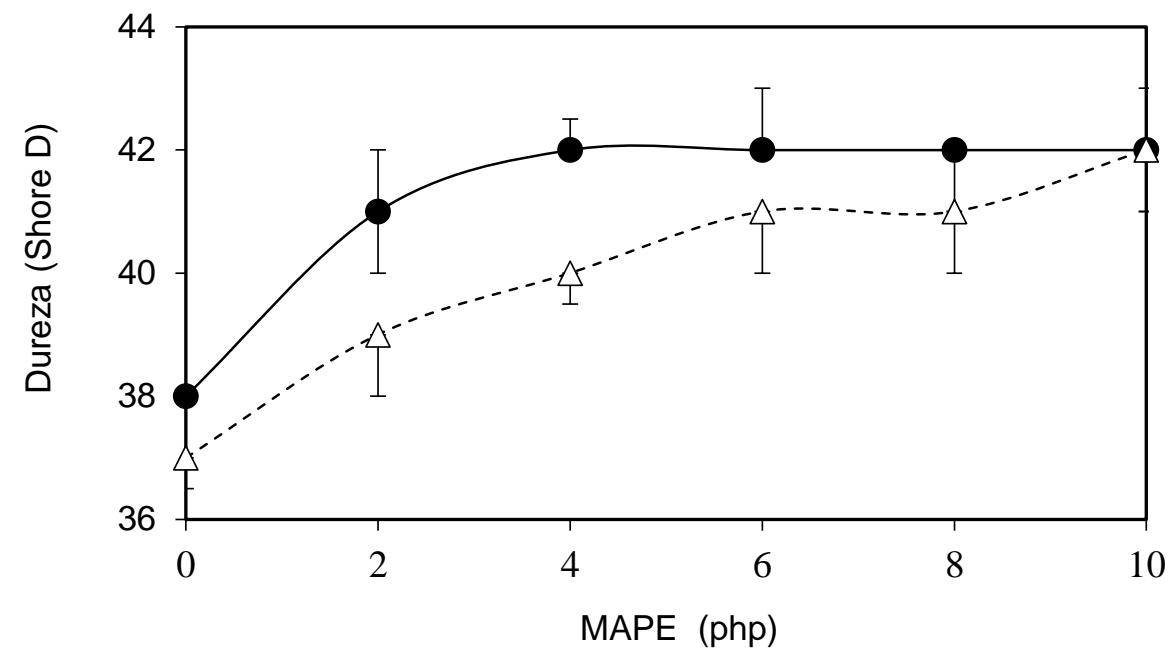

Fig. 6: Compuesto de EVA. Dureza. - - $\Delta$ - - HTM 40 php $\quad-\bullet-$ HTM 25 php.

\section{CONCLUSIONES}

La incorporación del MAPE como agente de acoplamiento en las mezclas, mejora la interacción harina polímero, permitiendo que la propagación de las tensiones en el material sea mucho más eficaz, hecho que se ve reflejado en el aumento de propiedades como el módulo de elasticidad, resistencia a la tracción y dureza de los compuestos de EVA, en comparación con aquellos que se formularon sin agente de acoplamiento.

El grado de unión alcanzado en la interface EVA - HTM, es consecuencia directa de la interacción entre los grupos hidroxilo de la celulosa en HTM, los grupos funcionales del MAPE y probablemente la naturaleza polar del EVA. Esta unión, sumada a la incorporación de la HTM, incrementa la probabilidad de encontrar puntos de falla en la inter-fase del compuesto.

Aunque la adición de la carga reforzante aumenta la rigidez y la resistencia a la tracción de los compuestos de EVA, a su vez reduce el volumen libre, restringiendo la movilidad de las cadenas poliméricas y afectando propiedades como la deformación y dureza.

En este caso particular, dada la naturaleza ligeramente polar del EVA, puede que se estén presentando problemas de compatibilidad entre EVA - MAPE y este hecho se pueda estar reflejando en las propiedades de tensión y dureza de los compuestos EVA/HTM/MAPE. 


\section{AGRADECIMIENTOS}

Este estudio forma parte del proyecto de investigación INV-ING-1546, el cual ha sido financiado por la Vicerrectoría de Investigaciones de la Universidad Militar Nueva Granada. Vigencia 2014.

\section{REFERENCIAS}

Arif, M. F., Megat-Yusoff, P. S. y Ahmad, F., Effects of Chemical Treatment on oil Palm Empty Fruit Bunch Reinforced High Density Polyethylene Composites, doi: 10.1177/0731684409348976, J. Reinf. Plast. Comp. 29(14), 2105 -2118 (2009)

ASTM.-“ASTM D 638-02 - Standard Test Method for Tensile Properties of Plastics" (2002)

ASTM.-“ASTM D2240-05 - Standard Test Method for Rubber Property—Durometer Hardness" (2010)

Bakar, N.A., Chee, C.Y., Abdullah, L.C., Ratnam, C.T. y Ibrahim, N.A. Thermal and Dynamic Mechanical Properties of Grafted Kenaf Filled Poly (vinyl Chloride)/ethylene Vinyl Acetate Composites, doi: 10.1016/j.matdes.2014.09.027, Mater. Design, 65, 204-211 (2015)

Bakar, N.A., Chee, C.Y., Abdullah, L.C., Ratnam, C.T. y Azowa, N. Effect of methyl methacrylate grafted kenaf on mechanical properties of polyvinyl chloride/ethylene vinyl acetate composites, doi: 10.1016/j.compositesa.2014.03.023, Compos. Part A-Appl. S. 63, 10-20 (2014)

Bengtsson, M. y Oksman, K. The use of silane technology in crosslinking polyethylene/wood flour composites, doi: 10.1016/j.compositesa.2005.06.014, Compos. Part A-Appl. S. 37(5), 752-765 (2006)

Bhattacharyya, A.R., Ghosh, A.K., Misra, A. y Eichhorn, K.J Reactively Compatibilised polyamide6/ethyleneCo-Vinyl Acetate Blends: Mechanical Properties and Morphology, doi: 10.1016/j.polymer.2004.12.012, Polymer, 46(5), 1661-1674 (2005)

Bhattacharyya, A.R., Ghosh, A.K. y Misra, A. Reactively Compatibilised Polymer Blends: A Case Study on PA6/EVA Blend System, doi: 10.1016/S0032-3861(01)00390-1, Polymer, 42(21), 9143-9154 (2001)

Dikobe, D.G. y Luyt, A.S. Morphology and Thermal Properties of Maleic Anhydride Grafted Polypropylene/ethylene-vinyl Acetate Copolymer/wood Powder Blend Composites, doi: 10.1002/app.31630, J. Appl. Polym. Sci. 116(6), 3193-3201 (2010)

Dikobe, D. G. y Luyt, A.S. Effect of Poly(ethylene-Co-Glycidyl Methacrylate) Compatibilizer Content on the Morphology and Physical Properties of Ethylene Vinyl Acetate-wood Fiber Composites, doi: 10.1002/app.26080, J. Appl. Polym. Sci. 104(5), 3206-3213 (2007)

Elanthikkal, S., Unnikrishnan, G., Varghese, S., James T. Guthrie, J.T. y Francis, T. Effect of Cellulose Whisker Content on the Properties of Poly(ethylene-Co-Vinyl Acetate)/cellulose Composites, doi: 10.1016/j.carbpol.2013.02.039, Carbohyd. Polym. 95(2), 773-779 (2013)

Faruk, O., Bledzki, A.K., Fink, H.P. y Sain, M. Biocomposites Reinforced with Natural Fibers: 2000-2010, doi: 10.1016/j.progpolymsci.2012.04.003, Prog. Polym. Sci. 37(11), 1552-1596 (2012)

Hwang, S.W., Ryu, H.C., Kim, S.W., Park, H.Y. y Seo, K.H. Grafting Maleic Anhydride onto EVA y Effect on the Physical y Rheological Properties of PETG/EVA-G-MAH Blends, doi: 10.1002/app.36592, J. Appl. Polym. Sci. 125(4), 2732-2739 (2012)

Kabir, M. M., Wang, H., Lau, K.T. y Cardona, F. Chemical Treatments on Plant-Based Natural Fibre Reinforced Polymer Composites: An Overview, doi: 10.1016/j.compositesb.2012.04.053, Compos. Part BEng., 43(7), 2883-2892 (2012)

Keener, T.J, Stuart, R.K. y Brown, T.K. Maleated Coupling Agents for Natural Fibre Composites, doi: 10.1016/j.compositesa.2003.09.014, Compos. Part A-Appl. S. 35(3), 357-362 (2004)

Kim, S.J., Shin, B.S., Hong, J.L., Cho, W.J. y Ha, C.S. Reactive Compatibilization of the PBT/EVA Blend by Maleic Anhydride, doi: 10.1016/S0032-3861(00)00810-7, Polymer 42(9), 4073-4080 (2001) 
Li, X., Tabil, L.G. y Panigrahi, S. Chemical Treatments of Natural Fiber for Use in Natural Fiber-Reinforced Composites: A Review, doi: 10.1007/s10924-006-0042-3, J. Polym. Env. 15(1), 25-33 (2007)

Ma, P., Hristova-Bogaerds, D.G., Schmit, P., Goossens, J.G. y Lemstra, P.J. Reactive Compatibilization of Ethylene-Co-Vinyl Acetate/starch Blends, doi: 10.1007/s13233-012-0153-8, Macromol. Res. 20(10), 1054$1062(2012)$

Madera-Santana, T.J., Soto-Valdez, H. y Richardson, M.O. Influence of Surface Treatments on the Physicochemical Properties of Short Sisal Fibers: Ethylene Vinyl Acetate Composites, doi: 10.1002/pen.23232, Polym. Eng. Sci. 53(1), 59-68 (2013)

Malunka, M.E., Luyt, A.S. y Krump, H. Preparation y Characterization of EVA-sisal Fiber Composites, doi: 10.1002/app.23650, J. Appl. Polym. Sci. 100(2), 1607-1617 (2006).

Navia, D.P., Ayala, A.A. y Villada, H.S. Biocompuestos de Harina de Yuca obtenidos por TermoCompresión. Efecto de las Condiciones de Proceso, doi: 10.4067/S0718-07642015000500008, Inf. Tecnol. 26(5), 55-62 (2015).

Onuegbu, G.C., Madufor, I.C. y Ogbobe, I. Studies on effect of maleated polyethylene compatibilizer on some mechanical properties of kola nut filled low density polyethylene,

htttp://www.savap.org.pk/journals/ARInt./ Vol.3(1)/2012(3.1-49).pdf. ISSN: 2223-9944, Acad. Res. Int. 3(1), 406-412 (2012)

Pracella, M., Haque, M. y Alvarez, V. Compatibilization and Properties of EVA Copolymers Containing Surface-Functionalized Cellulose Microfibers, doi: 10.1002/mame.201000175, Macromol. Mater. Eng. 295(10), 949-957 (2010)

Radhakrishnan, C. K., Kumari, P., Sujith, A. y Unnikrishnan, G. Dynamic Mechanical Properties of Styrene Butadiene Rubber and Poly (ethylene-Co-Vinyl Acetate) Blends, doi: 10.1007/s10965-007-9155-1, J. Polym. Res. 15(2), 161-171 (2007)

Rodríguez, L.J, William A. Sarache, W.A. y Orrego C.E. Compuestos de Poliéster Reforzados con Fibra de Plátano/Banano (Musa paradisiaca) Modificada Químicamente. Comparación con Fibra de Vidrio y Fique (Furcraea andina), doi: 10.4067/S0718-07642014000500005, Inf. Tecnol. 25(5), 27-34 (2014)

Salmah, H., Faisal, A. y Kamarudin, H. Chemical Modification of Chitosan Filled Polypropylene (PP) Composites: The Effect of 3-Aminopropyltriethoxysilane on Mechanical and Thermal Properties, doi: 10.1080/00914037.2010.531812, Int. J. Polym. Mater. 60(7), 429-440 (2011)

Silviya, E. K., Unnikrishnan, G., Varghese, S. y Guthrie, J.T. Thermal y Mechanical Characterization of EVA/banana Fiber-Derived Cellulose Composites, doi: 10.1002/app.35140 J. Appl. Polym. Sci. 125(1), 786$792(2012)$

Solis, M.E y Lisperguer, J.H. Resistencia al Impacto y a la Tracción de Materiales Compuestos PlásticoMadera, http://dx.doi.org/10.4067/S0718-07642005000600004, ISSN 0718-0764, Inf. Tecnol. 16(6), 21-25 (2005)

Sonia, A., K. Priya, D.K. y Alex, R. Celluloses Microfibres (CMF) Reinforced Poly (ethylene-Co-Vinyl Acetate) (EVA) Composites: Dynamic Mechanical, Gamma and Thermal Ageing Studies, doi: 10.1016/j.cej.2013.04.091, Chem. Eng. J. 228, 1214-1222 (2013)

Yang, H.S., Wolcott, M.P., Kim, H.S., Kim, S. y Kim, H.J. Effect of Different Compatibilizing Agents on the Mechanical Properties of Lignocellulosic Material Filled Polyethylene Bio-Composites, doi: 10.1016/j.compstruct.2006.02.016, Compos. Struct. 79(3), 369-375 (2007)

Yin, J., Zhang, J. y Yao, Y. Melt Grafting of Poly(ethylene-Vinyl Acetate) Copolymer with Maleic Anhydride, doi: 10.1002/app.24320 J. Appl. Polym. Sci. 102(1), 841-846 (2006)

Zimmermann, M.V., Turella, T.C., Santana, R.M. y Zattera, A.J. The Influence of Wood Flour Particle Size y Content on the Rheological, Physical, Mechanical and Morphological Properties of EVA/wood Cellular Composites, doi: 10.1016/j.matdes.2014.01.010, Mater. Design, 57, 660-666 (2014) 